\title{
Altersvorsorge von selbständigen Ärztinnen und Ärzten - die wichtigsten Änderungen der 1. BVG-Revision
}

Jean-Pierre Ceccon

Korrespondenz: Jean-Pierre Ceccon

Eidg. dipl. Finanzplanungsexperte

Financial Planner CFP ${ }^{\circledR}$

FMH Insurance Services

Baselstrasse 10

CH-4222 Zwingen

Tel. 0612610808

Fax 0612610805

jean-pierre.ceccon@fmhinsurance.ch
Das dritte und letzte Gesetzespaket der 1. BVGRevision hat per 1. Januar 2006 diverse, mitunter auch positive Änderungen in Steuerfragen gebracht. Diese betreffen insbesondere die Handhabung von Einkäufen in die 2. Säule.

\section{Einkäufe und Vorbezüge zwecks Wohneigentumsförderung (WEF)}

Wer für die Finanzierung seiner Immobilie Kapital aus der Pensionskasse bezogen hat, muss dies erst wieder zurückzahlen, bevor steuergünstige Einkäufe getätigt werden können. Wer solche Vorbezüge zwecks Finanzierung einer Immobilie plant, sollte sich von unabhängiger Seite gut beraten lassen. Die Erfahrung zeigt, dass geplante, steuergünstige Einkäufe in die Pensionskasse dann sinnvoll sind, wenn das Einkommen in einer hohen steuerlichen Progression steht - eine Progression von 40\% ist in manchen Kantonen bereits ab Fr. 150 000.- erreicht. Bevor Sie WEFVorbezüge tätigen, empfehlen wir Ihnen, sich in Anbetracht einer sinnvollen Steuerplanung beraten zu lassen.

\section{Kapitalbezug nach freiwilligem Einkauf}

Wer ab 2006 einen freiwilligen Einkauf vornimmt, kann die daraus resultierende Leistung innerhalb von drei Jahren vor dem Ruhestand nicht in Kapitalform beziehen. Unklar ist im Moment noch, für welchen Teil des Altersguthabens diese Restriktion gilt: für das gesamte Kapital, d.h. auch für den Teil, der vor den Einkäufen bereits bestand? Oder nur für den Teil, der neu eingekauft wurde? Ob dies auch für frühzeitig erstellte Freizügigkeitskonten oder eine separate Kadervorsorge gilt, ist ebenfalls noch unklar. In einer sorgfältigen Finanzplanung wird im Einzelfall die vorgeschlagene Lösung mit der Steuerbehörde abgeklärt.

\section{Einkaufslimitierung entfällt}

Tendenziell wurden durch die 1. BVG-Revision strengere Auflagen und Beschränkungen einge- führt. Anderseits erliess der Gesetzgeber auch Lockerungen von bestehenden Beschränkungen. Eine Lockerung betrifft die Einkaufslimitierung gem. Art. 79a BVG. Gemäss diesem Artikel wurde ein möglicher Einkauf auf die Anzahl Jahre bis zum Ruhestand, multipliziert mit Fr. 77400.-, limitiert. Neu besteht die einzige Limite in der Höhe des reglementarischen zulässigen Einkaufs der jeweiligen Pensionskasse und der darin gewählten Vorsorge.

\section{Anrechnung von Säule-3a-Guthaben}

Nach der bisherigen Methode wurden 80\% des vorhandenen Säule-3a-Guthabens als anrechenbar bestimmt. Basis für die neue Methode bildet eine Tabelle des Bundesamts für Sozialversicherung (BSV).

\section{Berechnungsbeispiel}

Bisherige Methode (gerundet)

Guthaben Säule 3a in Fr.

$355000 .-$

Davon anrechenbar in \%

$80 \%$

Davon anrechenbar in Fr. 284000.

Neue Methode nach Tabelle BSV (gerundet) Geburtsjahr

1963

Stand am 31.12.2005 in Fr.

Guthaben Säule 3a in Fr.

132000.-

Anteil kleine Säule 3a

355 000.-

Anrechenbar

132000 .-

223000.

Nach der neuen Methode besteht in diesem Beispiel eine Differenz von Fr. 61000.- zugunsten einer höheren Einkaufssumme.

\section{Vorfinanzierung der Frühpensionierung}

Nach der neuen Regelung beträgt das neue Pensionierungsalter 58 Jahre. Die fehlenden 7 Jahre bis Alter 65 können nun vorfinanziert werden. Dies ermöglicht weiteres Einkaufspotential. Wichtig: Wenn jemand über das vorfinanzierte vorzeitige Rücktrittsalter hinaus arbeitet, so muss 
die Vorsorgeeinrichtung dafür sorgen, dass das reglementarische Leistungsziel um nicht mehr als $5 \%$ überschritten wird.

\section{Fazit}

Richtig eingesetzt und geplant, ist die Pensionskasse nach wie vor das effizienteste Instrument zur Altersvorsorge.
Um die jeweils optimale Lösung zu bestimmen, empfiehlt es sich, dies durch einen ausgewiesenen Finanzplaner oder Vorsorgespezialisten berechnen zu lassen. Die Berater Ihrer standeseigenen Organisation «FMH Insurance Services» sind ausgewiesene und unabhängige Spezialisten.

\section{Steuererklärung ausfüllen - ohne Ärger und Stress}

Sie gehört sicherlich nicht zu den liebsten Aufgaben von Herrn und Frau Schweizer: das Ausfüllen der Steuererklärung. In den letzten Wochen wurden die Steuerpflichtigen wieder mit den entsprechenden leeren Formularen beglückt, welche jetzt nur darauf warten, ausgefüllt zu werden. Falls Sie das bereits getan haben, können Sie diesen Artikel getrost überspringen oder für das nächste Jahr auf die Seite legen. Ansonsten finden Sie nachfolgend einige Tips, damit Ihnen das Ausfüllen leichterfällt, und vor allem, wie Sie zu einer korrekten Veranlagung kommen - selbst wenn Sie Ihren Treuhänder oder Steuerberater mit dieser Aufgabe beauftragen.

Patrick Salathe

Korrespondenz:

Patrick Salathe

Dipl. Treuhandexperte

FMH Treuhand Services

c/o SALATHE TREUHAND AG

Ribigasse 3

CH-4434 Hölstein

Tel. 0619569100

Fax 0619569109

patrick.salathe@fmhtreuhand.ch
Wahrscheinlich nehmen auch Sie sich jedes Jahr wieder vor, der Steuererklärung einmal mehr Zeit zu widmen, die Wegleitung zu studieren und die Formulare wirklich gründlich auszufüllen. Als Lohn für Ihre Mühen erwarten Sie eine satte Steuerersparnis. Doch spätestens wenn der Abgabetermin näherrückt oder die bereits verlängerte Frist abläuft, gerät dieser Vorsatz wieder ins Wanken. In der Not nehmen Sie wie jedes Jahr die alte Steuererklärung als Vorlage hervor und setzen die neuen Zahlen ein. Und dann nichts wie weg damit!

Leider birgt dieses Vorgehen erhebliche Risiken, denn sowohl Ihre Lebensumstände wie auch die Steuerlandschaft ändern sich laufend. Und wenn Sie bisher wichtige Abzüge vergessen haben, werden Sie diese nach obiger Methode mit Sicherheit auch in Zukunft vergessen. Dabei ist eine korrekte Steuerdeklaration gar nicht so eine Hexerei, wenn man sich an einige einfache Grundsätze hält - und die wichtigsten Fehler vermeidet, die sich leider in der Praxis immer wieder zeigen.

\section{Das Ausfüllen beginnt bereits ein Jahr früher!}

Die mühsamste Aufgabe ist nämlich meist das Sammeln aller nötigen Belege und Beilagen. Richten Sie sich daher ein Dauermäppchen oder -ordner speziell für die Steuerunterlagen ein. Dort kommen übers Jahr alle Belege hinein, die steuerlich relevant sind. Und zwar noch bevor sie irgendwo im Schreibtisch oder in einer anderen Ablage verschwinden. Ehe Sie sich dann an die Steuererklärung wagen, sollten Sie mit Hilfe der ausgefüllten Steuererklärung der Vorperiode die Belege auf Vollständigkeit prüfen. Gibt es auch in diesem Jahr zu jedem letztjährigen Posten einen Beleg? Um sicherzustellen, dass Sie auch nichts Neues vergessen, das in Ihrer letzten Steuererklärung noch nicht deklariert worden ist, lohnt sich folgendes Vorgehen: Gehen Sie das aktuelle, leere Steuererklärungshauptformular Posten für Posten durch, und überlegen Sie sich, welche der Posten für Sie bzw. Ihre Familie im vergangenen Jahr relevant waren. Im Zweifelsfall können Sie auch noch die Wegleitung konsultie- 
ren. Leider stellt nur dieses auf den ersten Blick etwas beschwerliche Vorgehen sicher, dass Sie nichts Wichtiges vergessen haben. Sollten Belege fehlen, können Sie diese jetzt noch besorgen.

Die beschriebenen Schritte sollten Sie übrigens auch durchgehen, wenn Sie die Steuererklärung vom Fachmann ausfüllen lassen. Denn der Fachmann kann seine Arbeit nur so gut machen, wie Sie ihm diese vorbereiten.

\section{Das Ausfüllen selbst wird ungemein erleichtert}

Haben Sie erst alle Unterlagen vollständig beisammen und sich mit den Änderungen in der Wegleitung vertraut gemacht, ist das eigentliche Ausfüllen der Steuererklärung keine grosse Übung mehr. Ob von Hand oder mittels EDV: Füllen Sie zuerst die Hilfs- und Beiblätter aus und arbeiten Sie sich dann zum Hauptformular vor. Ohne EDV benutzt man am besten zuerst einmal das Doppel. Erst wenn Sie nochmals alle Ihre Eingaben mit den Belegen abgestimmt, Ihre Rechenoperationen nachgerechnet und die steuerliche Vermögensentwicklung abgecheckt haben, übertragen Sie die Daten auf das Hauptformular. Fertig!

\section{Ein Wort zur steuerlichen Vermögensentwicklung}

Leider kontrollieren die wenigsten Steuerpflichtigen ihre steuerliche Vermögensentwicklung. Die Steuerbehörden tun dies aber sehr wohl und stellen auf diese Weise Fehler in den Deklarationen fest. Ungereimtheiten in der Vermögensentwicklung deuten nämlich auf fehlende oder falsche Angaben hin. Mit Vorteil kontrollieren Sie diese daher selbst nach folgendem Schema:

$\begin{aligned} & \text { Steuerbares Vermögen } \\ & \text { laut aktueller Steuererklärung }\end{aligned}$
$\begin{aligned} & \text { - steuerbares Vermögen } \\ & \text { am Ende der Vorperiode }\end{aligned}$
$\begin{array}{ll}\text { Vermögenszu- oder -abnahme } & \text { Fr. }\end{array}$

Die Vermögenszu- oder -abnahme wird nun in Relation zum Einkommen und zu den Ausgaben gesetzt:

$\begin{array}{ll}\text { Einkommen } & \text { Fr. } \\ \begin{array}{l}\text { - Ausgaben gemäss Abzügen } \\ \text { der Steuererklärung }\end{array} & \text { Fr. } \\ \text { - Ausgaben für Lebensunterhalt (Schätzung) } & \text { Fr. } \\ \pm \text { Sonderfaktoren (siehe unten) } & \text { Fr. } \\ \begin{array}{l}\text { Muss etwa der Vermögenszu- } \\ \text { oder -abnahme entsprechen }\end{array} & \text { Fr. }\end{array}$

Als Sonderfaktoren kommen auch bei korrekter Deklaration zum Beispiel folgende Transaktionen in Frage: Käufe und Verkäufe von Liegenschaften (wegen der Differenz zwischen Steuer- und Verkehrswert), Schenkungen oder Erbschaften, Kapitalgewinne an der Börse, Bewertungsdifferenzen zwischen steuerlichem Wertschriftenverzeichnis und effektivem Wert, Verkäufe von privaten Gegenständen, Investitionen in Liegenschaften usw.

\section{Diese Fehler passieren häufig}

Erfahrungsgemäss werden oft kleinere Nebeneinkünfte, Einkommen aus dem Ausland sowie Kapitalabfindungen und Vorbezüge von Vorsorgeleistungen vergessen zu deklarieren. Bei den Abzügen sind die Steuerpflichtigen vielfach bei speziellen Berufsauslagen, Schulden/Schuldzinsen (auch von kleineren Schuldbeträgen wie Kreditkarten usw.), Krankheits- und Invaliditätskosten sowie freiwilligen Zuwendungen nachlässig. Viel Geld wird verschenkt, wenn die Unkosten für Renovationen der Liegenschaft nicht angegeben oder falsch geplant werden. Hier lohnt sich übrigens bei grösseren Renovationen genau wie bei Einkäufen in die 2. Säule eine gescheite zeitliche Planung.

Beim Vermögen gehen schnell einmal saldierte Bankkonti, rückkaufsfähige Lebensversicherungen (nicht jedoch die der gebundenen 3. Säule), Vermögenswerte wie Zweitfahrzeuge, Barschaften, Edelmetalle und Schmuck, Sammlungen und andere mehr oder weniger teure «Hobbyartikel» wie Motorräder, Oldtimer, Wohnwagen/ Camper, Yachten, Sportflugzeuge etc. vergessen. Gerade bei Hobby und Freizeit denkt man nämlich kaum an die Steuern. So gehört freilich auch das Ferienhaus oder die Ferienwohnung deklariert, selbst wenn im Ausland gelegen.

Einen Topplatz auf der Liste der nicht deklarierten Werte haben Schenkungen (erhaltene oder gemachte) und Erbschaften. Letztere gehören auch aufgeführt, wenn diese schon vor Jahren angefallen sind, aber die Verteilung noch nicht stattgefunden hat (Anteile an Erbengemeinschaften: Einkommens- und Vermögensanteil sind zu deklarieren).

Ein Problem entsteht vor allem dann, wenn nicht deklarierte Vermögenswerte veräussert werden. Verkauft man nach ein paar Jahren sein Ferienhaus in Südfrankreich wieder, fliesst einem plötzlich steuerlich nicht erfasstes Vermögen zu. Schlimmer als eine allfällige Nachsteuer auf dem nicht deklarierten Vermögen ist die Tatsache, dass der Fiskus von Ihnen unter Umständen wissen will, wie Sie diese Liegenschaft dazumal 
finanziert haben. Fehlt ein vernünftiger Nachweis, können die Steuerbehörden sehr unangenehm werden.

\section{Verhalten gegenüber den Steuerbehörden}

Da Steuerbeamte bekanntlich auch nur Menschen sind, möchten diese auch als solche behandelt werden. Dies gilt bei allfälligen Rückfragen genauso wie schon beim Ausfüllen der Steuererklärung. Wer den Steuerkommissär mit masslosen Abzügen provoziert, muss damit rechnen, dass es dieser besonders genau nimmt. Ebenso erleichtern Sie dem Steuerbeamten mit vollständigen, plausiblen und sauberen Angaben die Arbeit, was er oder sie Ihnen hoffentlich mit einer reibungslosen und wohlwollenden Steuerveranlagung dankt.

Und genau diese sollten Sie unbedingt kontrollieren. Denn leider unterlaufen auch den Steuerämtern immer wieder Fehler, oder es werden Aufrechnungen vorgenommen, die nicht in Ihrem Sinne sind. Dann sollten Sie innerhalb der Einsprachefrist unbedingt auch Einsprache erheben. Denn hier gilt im allgemeinen: verpasste Frist $=$ verwirktes Recht!

\section{Nicht in Versuchung geraten}

Es ist nur menschlich, dass man alles Mögliche tut, um Steuern zu sparen. Daher sollten jegliche Ermessensspielräume ausgenutzt werden. Aber beim Ausnutzen sollte es dann auch bleiben. Werden die Grenzen nämlich überschritten, kann einen das sehr teuer zu stehen kommen. Das nennt der Steuervogt dann versuchte oder vollendete Steuerhinterziehung. Sollte noch die eigenmächtige «Änderung» von Belegen im Spiel sein, wirft einem der Fiskus so unangenehme Dinge wie Steuerbetrug und/oder Urkundenfälschung vor. Das ist dann kein Kavaliersdelikt mehr und hat strafrechtliche Konsequenzen!

Ganz allgemein gilt: Steuerbeamte sind nicht auf den Kopf gefallen. Sie haben vielfältige Kontrollmöglichkeiten und ein ausgeklügeltes System von Quervergleichen und Meldungen geschaffen, um eine korrekte Steuerveranlagung zu erzwingen. So kann eine Kontonummer auf einem eingereichten Auszahlungsbeleg einen
Hinweis auf ein nicht deklariertes Konto geben. Oder eine Versicherung meldet eine Leistung direkt dem Fiskus. Allenfalls stellt die Steuerverwaltung bei einer Steuerrevision einer Unternehmung Tatsachen fest, die genau Sie betreffen, und geht der Spur nach. Vielleicht ist auch nur die obenerwähnte Vermögensentwicklung nicht plausibel. Es lohnt sich auf jeden Fall, bei der Wahrheit zu bleiben.

\section{Steuerberater, Treuhänder \& Co.}

Möglicherweise kommen Sie auch zur Auffassung, dass sich ein Fachmann um Ihre Steuerangelegenheiten kümmern sollte. Das ist oft sinnvoll und drängt sich in komplexeren Verhältnissen - wenn einem das nötige Spezialwissen fehlt - geradezu auf.

In diesem Zusammenhang ist es jedoch nicht nur wichtig, sich an den richtigen Berater (Kompetenznachweis durch entsprechendes Diplom und/oder langjährige Erfahrung, guter Ruf, Verbandszugehörigkeit usw.) zu wenden, sondern auch, diesem alle nötigen Unterlagen und Informationen zur Verfügung zu stellen. Sonst nützt Ihnen der beste Fachmann wenig. Deshalb kommt auch der Vollständigkeit der Belege eine so zentrale Bedeutung zu.

Ebenfalls wichtig ist, dass Sie Ihrem Berater die definitive Steuerveranlagung rechtzeitig zur Kontrolle zukommen lassen, falls es Abweichungen zur Deklaration gibt. Ihr Berater muss noch genügend Zeit haben, um gegebenenfalls Einsprache erheben zu können.

Und ganz allgemein gilt: Seien Sie ruhig etwas kritisch, stellen Sie Fragen, und versuchen Sie, die Arbeit Ihres Beraters nachzuvollziehen.

\section{Fazit}

Das Ausfüllen der Steuererklärung wird meist im Kopf zu einer schwierigeren und unangenehmeren Aufgabe aufgebläht, als sie eigentlich wäre. Wenn Sie obigen einfachen Punkten Beachtung schenken, werden Sie mit Sicherheit der nächsten Steuererklärung und auch der Steuerrechnung gelassener entgegenschauen. Denn Sie wissen, dass Sie alles korrekt ausgefüllt und für sich das Optimum herausgeholt haben. 